\title{
REMOTE OPERATIONS IN A GLOBAL ACCELERATOR NETWORK
}

\author{
S. Peggs, T. Satogata, BNL, USA, D. Agarwal, LBNL, USA, D. Rice, Cornell University, USA
}

\section{Abstract}

The INTRODUCTION to this paper summarizes the history of the Global Accelerator Network (GAN) concept and the recent workshops that discussed the relationship between GAN and Remote Operations. The REMOTE OPERATIONS SCENARIOS section brings out the organizational philosophy embodied in GAN-like and to non-GANlike scenarios. The set of major TOPICS RAISED AT THE WORKSHOPS are only partially resolved. COLLABORATION TOOLS are described and discussed, followed by examples of REMOTE ACCELERATOR CONTROL PROJECTS around the world.

\section{INTRODUCTION}

Remote control of accelerators has been widespread for about 2 decades, in large accelerators such as LEP and HERA where the control room is of order $10 \mathrm{~km}$ from the farthest part of the ring. Accelerator engineers and physicists routinely "dial in" to lab computers to check on their equipment, or for general adjustments to equipment and databases, where security policies permit. Technically, most of these duties could be done just as well $1,000 \mathrm{~km}$ from the accelerator. The arrival of ever higher bandwidth connections, in the home, office, and in the lab, promises to greatly enhance such routine "single site" remote operations. Recently, much interest has been expressed in "multiple site" remote operations. Although it is not necessary to tightly link the two topics, "Remote Operations" and "GAN" are often considered together. While the original GAN inspiration was the goal of enabling construction of a Linear Collider as an international project, the same remote operations concepts can be applied to any accelerator, large or small, trans-national or intra-national, new or old. Multiple site remote operations are potentially relevant to the APS, CESR, LHC, and the VLHC, as well as to a Linear Collider.

Reports and workshops. Two ICFA working groups studied and reported on the GAN concept in 2001 [1]. The first report examines the General Considerations and Implementation, while the second reports on many Technical Considerations that are relevant to the topic at hand, Remote Operations. Three workshops were held in 2002, gathering together social scientists and members of the accelerator and experimental community, to consider "Enabling the Global Accelerator Network" (Cornell University), "Collaboration Tools for the Global Accelerator Network" (Lawrence Berkeley Laboratory), and "Remote Operations" (Shelter Island) $[2,3,4]$.

REAP. Early in 2003 a working group on Remote Experiments in Accelerator Physics [5] (REAP) was formed un- der the Beam Dynamics Panel [6] of ICFA, to promote and facilitate communication between laboratories interested in accelerator remote operations. While the focus is on accelerator physics experiments, REAP activities share many of the technical and sociological challenges that a GAN would face. Lessons learned in carrying out remote accelerator physics experiments will be invaluable in setting up more extensive remote operations. REAP maintains a database of accelerator physics experiments employing remote operations, and is developing guidelines for easily accessible webcast seminars. Future work also includes maintaining a library of documentation on remote operations, distributing newsletters, further development of network communications, and workshop sponsorship.

Foundation technologies. The accelerator and experimental physies communities were crucial early implementers of the World Wide Web foundation technology, both because of our extreme performance requirements, and because of our willingness and ability to work at the bleeding edge. It is reasonable - although far from certain - to expect that we will again be involved in a profound paradigm shift, through the emerging technologies that will enable us to meet the rapidly expanding challenges of experimental data analysis, and Remote Operations. Even less certain is the timescale, the exact implementation, and, especially, the social impact of the next great leap forward. Consider, for example, the potential impact of Remote Operations in the context of pilot-less military airplanes.

The need to greatly enhance our ability to share resources in real time increases by orders of magnitude the demands that are placed on networks. Remote control rooms and video conferencing are examples of functions that place such demands - what will they look like with 3 or 6 more orders of magnitude of bandwidth?

\section{REMOTE OPERATIONS SCENARIOS}

Experience with multi-institution accelerator construction projects, and particle physics experiments, illuminates the challenges in balancing the need for partner institution autonomy against the need for successful and efficient integration. However, collaborative accelerator construction projects to date have employed the "build and forget" management model - responsibility and ownership is sooner or later handed off completely to the "host laboratory". (This is not the case for particle physics experiments, where the collaborations continue on a more or less equal footing well beyond the construction and data-taking phases.) In contrast, the central tenet of the GAN philosophy is that the partner laboratories remain involved in perpetuity, as equal partners. There is no "host laboratory" but rather a "site 
laboratory", and the Project Manager (for example) is probably not an employee of the site laboratory. That is, the site laboratory relinquishes centralized control, in order to secure the continued distributed symmetric participation of the partners who build the accelerator sub-systems.

The need for a distributed symmetric organization is probably the most important example of a high level external requirement in a GAN-like remote operations scenario. However, there are many other new issues - technical, social, and non-traditional - that are also vitally important to both GAN-like and non-GAN-like scenarios. Needs that must be addressed in any remote operations scenario include facility coordination and intimate day-today facility knowledge, emergency response, and procedural consistency. New challenges potentially include crosscultural integration, language adoption, and the lack of casual, serendipitous interactions. These challenges are often organizational and social, rather than technical.

Many of these same issues are also relevant, although perhaps not so stressful, to the experimental physics community. For example, the CMS experiment in the LHC plans to install a Virtual Control Room at Fermilab. Some take the GAN philosophy one step further, in proposing the concept of a joint accelerator/experimental Virtual Control Room.

\section{Symmetric and Asymmetric Control Rooms}

Potential remote control room implementations cover a spectrum from a simple asymmetric scenario with occasional consultation of remote experts, to a symmetric scenario with functionally- and organizationally-equivalent control rooms at each partner laboratory. In the symmetric sequential model the executive privilege for operating the accelerator is passed off between multiple control rooms that are (close to) identical. Control of the accelerator complex rotates between the partner labs, perhaps as quickly as shift by shift. In the symmetric simultaneous model, multiple control rooms are simultaneously all more or less equally active, nonethetess with executive control clearly defined. A control room in an asymmetric scenario may be complete, or it may invoke collaborative tools to give just a virtual presence in a complete on-site control room. The functionality can then be adjusted to best serve the diverse needs of each remote operations user group.

The needs of many operations aser groups must be considered during routine and non-routine operations. These groups include operation crews and co-ordinators, accelerator physicists, experimental groups, subsystem hardware and software experts, application programmers, control system integration experts, and system administrators. While there is no simple consensus on a best remote operations scenario for all these groups, there is a general consensus that any remote experts responsible for a subsystem must remain actively engaged, through continued accelerator operations. There is also a consensus that an off-site control room with full remote operations capabil- ity is now (or soon will be) a feasible alternative to routine travel to the site.

\section{TOPICS RAISED AT THE WORKSHOPS}

Details of the workshop discussions on the following topics can be found in the 3 workshop proceedings $[2,3,4]$.

Controls Architecture. Remote operations, like large international detector construction, sets critical consistency constraints on controls language, training, and procedures. Comprehensive system simulations and early adoption of top-and middleware tools are essential, and standards must be followed to assure facility consistency and maintainability. This is particularly important in the project's system integration and commissioning phases. Security is a particularly important part of the controls architecture.

Standardization versus accommodation. Standards are organizationally required for language, software development and version control, documents, testing, quality assurance and operations procedures. Negotiation of the balance between a strong central authority responsible for integration, and diverse partner labs responsible for development, operations and support, is among the dominant challenges of large-scale remote operations. How far through the control engineering systems must uniformity be required? Uniformity leads to lower costs, better maintainability, and decreased confusion, but accommodation leads to design flexibility and a greater degree of "ownership". A standards committee should urge conformity, without rushing to establish standards prematurely.

Operational evolution. Installation and commissioning require a substantial on-site presence of participants, including future remote experts. This period is a primary opportunity for community and mutual interest building that will sustain interest through the transition from early commissioning to the continuous upgrade phase. The remote operations environment must be attractive to the operations user groups, in order to keep activity levels high.

Social commumications. For long-term organizational viability, remote operations should allow nearly the same range of interpersonal interactions as co-located personnel. Easily used communication devices such as video walls should proliferate. Casual interactions and other low-level contacts are the most important channels for team-building - familiarity breeds content.

Training and procedures. Common operator training is required. Inter-lab participatory tutelage and "hands-on" training will also build community and encourage common practices, but early simulation training will potentially be quite difficult. All operations documentation and reviews must be freely-available from all control rooms, including playback of operations activities for training and review purposes. Routine mixing of operations member assignments between member labs will be useful to promote community.

Maintenance and spares. Remote maintenance scenarios include the need for detailed consultation between an 
on-site repair crew and remote experts. Ownership and responsibility for failed components must be clearly defined, and there must be checks and balances for remote experts to verify repairs and proper system performance. It is not completely obvious that an increased spares inventory is necessary, especially if components are duplicated among multiple projects supported by an individual participant laboratory.

The 1\% problem. Expert intervention on-site is expected about 20 times per year, or for about $1 \%$ of interventions, in a large remote operations facility. This typically implies an interruption of operations for approximately two days if extended air travel is required. Possible remedies include improved internal diagnostics, extensive logging, and enhanced remote diagnosis. None of these solutions are prohibitively expensive, and all of them are also "good practice" features for a locally-controlled accelerator.

An Example: KEK ATF. After careful consideration in 2000 , KEK personnel decided not to retroactively implement a full remote operations capability for the ATF ring. The radiation safety reporting path was critical in this decision - an on-site control room shift leader is required for all shifts. Most of the appropriate documentation was in Japanese, providing a language barrier to foreign collaboration. The technical problems were surmountable, but inbuilt social and organizational issues demonstrated that remote operations as an afterthought is at best difficult.

\section{COLLABORATION TOOLS}

Many efforts to build and deploy collaboration environments in support of remote access have been launched in the past decade. As a result, many collaborative tools are now available, and several studies of collaborations have been conducted. Success depends on many factors including the technologies used, the social environment, the goals, the level of support, and the level of need. An important factor is time and place: users might work at the same or different times, and in the same or different places. Collaboration often involves a mixture of types of interactions, requiring an appropriate array of tools.

\section{Technologies}

Collaboration technologies facilitate several modes of interaction. One mode involves interpersonal communication including speech, video, text, and e-mail, while another enables access to stored data and information, including real time monitoring. Several tools support conversations. Text-based chats like Instant Messaging (IM), Multi-User Dungeons (MUD), and Internet Relay Chats (IRC) provide mostly synchronous interaction but can also allow intermittent interactions. Secure text messaging and presence capabilities are provided by the Berkeley Lab Secure Messaging Tool. Video conferencing tools include Polycom Video Conferencing, NetMeeting, Virtual Rooms Videoconferencing System (VRVS), and Access Grids. These tools provide varying degrees of visual and audio interaction capabilities and immersion. The Access Grid provides a very immersive experience with a large video wall to project all the participants, several camera views of participants, and naturalistic audio. Technologies like NetMeeting, Via Video, and the VRVS system provide limited immersion capabilities during workstationbased interactions, including meetings over high and low bandwidth. Capture and replay capabilities are currently emerging. Soon, data will be archived and annotated for continuing discussion.

Several tools augment synchronous interactions, allowing participants to talk about a shared display, data stream, or report. A number of technologies allow this: screen sharing, electronic whiteboards, presentation software, and remote control panels. Ideas currently under development include workstation "docking" and peer-to-peer file sharing capabilities, which allow users to share data on any computer or PDA in a relatively ad hoc fashion. Electronic notebooks that allow access to an organized stream of activity, comments, and data are in use in various communities. Most accelerators already use electronic log-books.

Some cutting edge technologies focus on the awareness that people have of others availability and current work. Presence information plays an important role in the perception that collaborators are working together. Collaborative workflow systems under development incorporate Grid technologies to provide security and to allow submission of compute jobs on Grid enabled machines. Shared editing of text and documents is an important collaborative activity that has not yet been adequately addressed.

\section{Social factors}

A number of issues - security, privacy, interruptions, ease of use and training - must all be addressed, before collaboration technologies are acceptable to end users. Participants need mechanisms for identifying themselves and having private interactions. A person on video wants to be able to see all the people viewing that video. Whatever technology is employed needs to be easy to use, and must be platform independent. Applications must be customizable to particular situations. Participants need to evolve interaction rules of conduct that are mutually acceptable and widely known.

Participants in collaborations across national and cultural boundaries (including between laboratories in the same country) may have to relinquish a substantial portion of their habits, to seek a common working mode. Conscious effort will have to be spent on finding best working practices, and ways to adapt to them. Semi-formal techniques such as "User-Centered Design" can be used to extract requirements for a collaborative environment by producing a preliminary view of the users goals, work practices, and likely interaction patterns and needs 


\section{Coordination functionalities}

The coordination that takes place in all these settings is both asynchronous and synchronous, involving both scheduled meetings and informal communication. Required functionalities include:

Meeting support. Users want to be able to give and attend meetings and presentations remotely, with both audio and video of the presenter along with the slides or presentations of other things (like a data stream, simulation results, 3-D CAD tools, video, documents, visualization and electronic notebooks). All participants should be able to see all other participants, in order to capture their reactions. People should be able to connect from their offices as well as from special conferencing rooms, and should be able to participate when traveling. This raises the issue of whether and how to make solitary participants equal members of meetings. If passive participation and multi-tasking is appropriate - perhaps when connected at the desktop - it is desirable to have the stream running in the background.

Informal meetings. On occasion, such as when an unexpected result happens, people need immediate access to associates for consultation. For this, an awareness/presence system with appropriate rules about its use (a cultural issue) will support finding and contacting the right person. Once contacted, the kinds of technology that support formal meetings would support these less formal sessions.

Remote operations. In order to establish efficient twoway communication it is only necessary to duplicate what is on a subset of screens, and NOT the entire control room. Screens should "look" the same to everyone. A meeting room is needed for the development and discussion of run plans, analysis, information exchange at shift changes, et cetera. Also needed are electronic whiteboards visible at remote locations, and computers for logging data streams, and for analyzing and displaying summary data. Since the operators hands and eyes are busy, the primary communication will likely be a wireless headset. Video of the remote partner helps communication. The same kind of views and communication channels help during installation, testing, and commissioning, et cetera, for those who need never be on site.

Experience at BNL/RHIC. A VRVS video conferencing system was used to broadcast shift change and other daily meetings during the 2003 RHIC run. Experiment control rooms and personnel offices had non-interactive access to these broadcasts. This approach was abandoned after approximately two weeks of use - only one operations coordinator knew how to operate the video conferencing equipment, and interest quickly waned when equipment problems developed. This experience emphasizes an adoption expectation for communications utilities: they should be as convenient and reliable as a telephone. Simple video conferencing systems are just barely reaching this state. Advanced video conferencing software like the Access Grid are far from this ease of use.

\section{ACCELERATOR REMOTE CONTROL PROJECTS}

A total of 22 in-progress and planned experiments was presented at the Shelter Island workshop, and is tabulated in the proceedings [7]. Table 1 lists a representative sample of six of them. A brief description of three remote operations activities (two of them initiated after the Shelter Island workshop) illustrates the breadth of these endeavors.

\section{Examples of current and planned experiments}

Small scale (current): Remote controls enable both faculty and students at a small university to experience and carry out research at a large facility, without frequent or prolonged travel. A series of remote diagnostic and tuning experiments on the Cornell Electron Storage Ring (CESR) is being carried out by a faculty member at Alfred University, a small institution approximately $100 \mathrm{~km}$ from the lab [8]. The faculty member had been a member of the accelerator physics staff at CESR for several years, and thus did not need to make an initial on-site stay to become familiar with accelerator infrastructure, or to build the personal links necessary for close collaboration. Orbit and aperture measurements on the injector synchrotron have been made.

Since the CESR control programs use X-windows displays, remote operation is enabled simply by logging in to the CESR control computers. (Additional control for authorization must be put in place before using the system on a widespread scale.) In addition to conventional control and monitoring functions (including graphical history displays) a digitizing oscilloscope provides updates of screen data at roughly $5 \mathrm{~Hz}$. Coordination with the accelerator operator is made by telephone.

Medium scale (current): An experiment to characterize and improve photoinjector performance has been in progress in the A0 hall at Fermilab for over 4 years [9]. The collaboration - between institutions in the US, Italy, and Germany - is working on development of an injector for the TESLA and TTF accelerators, and on the study of novel applications of high brightness, pulsed electron beams. For example, a recent study of the effect of the injectors bunch compression chicane on bunch properties has been carried out primarily by operation from DESY $[10,11]$. The central energy, energy spread, bunch length, and transverse emittance were measured for several combinations of RF cavity phase and chicane magnet currents.

Because the photoinjector experiment was not originally designed with remote operation in mind, several functions - RF transmitter on/off, laser adjustments, and cryogenics control - are performed locally. These need only intermittent attention, mostly at the beginning and end of shifts. Other local functions are also available remotely using VNC, a cross platform program duplicating a local computer screen and keyboard at a remote site. A remotely controlled video switch allows any of a number of TV images to be sent to a web browser window faster than $1 \mathrm{~Hz}$. The composite signal is captured by a frame grabber, then 
Table 1: Representative Remote Operations Projects

\begin{tabular}{|c|c|c|c|c|c|}
\hline Partners & Experiment & Goals & Site Lab & Software & Status \\
\hline BNL, FNAL & IR Linear Corr. & Remote beam ops & $\begin{array}{l}\text { BNL } \\
\text { (RHIC) }\end{array}$ & $\begin{array}{l}\text { Local, } \\
\text { ACNET }\end{array}$ & Proposed \\
\hline JLab, SNS, LANL & $\begin{array}{l}\text { SNS SRF } \\
\text { Commissioning }\end{array}$ & Evaluate RF cavity perf. & JLab & EPICS & Proposed \\
\hline $\begin{array}{l}\text { DESY, Cornell, } \\
\text { Ohio State, U. Mich. }\end{array}$ & TTF DAQ & $\begin{array}{l}\text { Database tools for accel. } \\
\text { data, collaborative tools }\end{array}$ & $\begin{array}{l}\text { DESY } \\
\text { (TTF) }\end{array}$ & $\begin{array}{l}\text { DOOCS, } \\
\text { VRVS }\end{array}$ & Proposed \\
\hline Cornell, Alfred U. & $\begin{array}{l}\text { Accelerator } \\
\text { diagnostics }\end{array}$ & $\begin{array}{l}\text { Devel. remote tools, } \\
\text { improve accel. perform. }\end{array}$ & $\begin{array}{l}\text { Cornell } \\
\text { (CESR) }\end{array}$ & $\begin{array}{l}\text { Local, } \\
\text { X-windows }\end{array}$ & Ongoing \\
\hline FNAL, DESY & A0 Photoinjector & $\begin{array}{l}\text { Develop high } \\
\text { current injectors }\end{array}$ & FNAL & $\begin{array}{l}\text { PC, MAC, } \\
\text { SUN }\end{array}$ & Ongoing \\
\hline KEK, SLAC & $\begin{array}{l}\text { Beam based } \\
\text { alignment and } \\
\text { analysis - ATF }\end{array}$ & $\begin{array}{l}\text { Damping ring } \\
\text { development, } \\
\text { remote control }\end{array}$ & $\begin{array}{l}\text { KEK } \\
\text { (ATF) }\end{array}$ & $\begin{array}{l}\text { MATLAB, } \\
\text { V-system }\end{array}$ & Ongoing \\
\hline
\end{tabular}

converted to jpeg format to reduce required bandwidth. Video conferencing and a mutually accessible electronic log book complete the remote control package.

Large seale (proposed): A recent proposal to the European Steering Group on Accelerator R\&D (ESGARD) [12] includes a comprehensive program to use working examples of a Multipurpose Virtual Laboratory (MVL) to demonstrate "far remote operation" capabilities and the essential components envisaged in GAN [13]. Seven different accelerator technology projects will be used as development test beds. The GANMVL collaboration of 6 major European laboratories plans a 3 year time scale for the project, with an estimated effort of 80 person-years.

MVL works in a client-server configuration. The server will contain hardware and software to capture measured accelerator data, user controls and audio/video data. These real-time data will be distributed to the clients and projected into an interactive virtual environment. In order to approach the scientific and social interaction environment of a single laboratory, video and audio links will use 3-D audio and stereo video technology. Streaming transmissions will employ secure technologies to guarantee smooth and uninterrupted data flow. Standardization of vocabulary, comprehensive authorization procedures, plug and play hardware, and planning for remote coordination of operations planning, maintenance, troubleshooting and repair will be studied and developed.

\section{CONCLUSIONS}

Multiple site remote operations are necessary in the GAN management model of the distributed symmetric participation of equal partners in a future large scale accelerator project. Remote operations - whether in a symmetric sequential, symmetric simuitaneous, or an asymmetric scenario - are also acquiring powerful potential advantages in non-GAN-like applications. Thanks to rapid communications advances, it is becoming possible to envisage tightly knit but broadly dispersed communities of accelerator op- erations user groups. The challenges to successful implementation are as much social as technical. Evolving technologies from the Access Grid to the Multipurpose Virtual Laboratory deserve close attention. However, new modes of communication must become as simple and reliable as a telephone before broad acceptance is assured. Contemporary video conferencing still leaves much to be desired. The broader societal impact of any such new foundation technology is as hard to predict as it was for the World Wide Web in its early evolutionary period.

\section{ACKNOWLEDGEMENTS}

We are very grateful for the contributions of K.Desler, F.Willeke, all the workshop participants, and the personnel of the CREW project at the University of Michigan.

\section{REFERENCES}

[1] http//www.final.gov/directorate/icfa/icfa_tforce_reports.html

[2] http://www.Ins.comell.edu/ganwkshp

[3] http://www-itg.lbl.gov/Collaboratories/GANMtg

[4] http:/www.rhichome.bnl.gov/RemOp

[5] http://www.lepp.cornell.edu/icfa/reap

[6] http://wwwslap.cern.ch/icfa/

[7] http:/www.rhichome.bnl.gov/RemOp/docs/ WG1_ganprojects_020919.PDF

[8] R. Holtzapple, private communication

[9] J.-P. Cameiro, et al, "First Results of the Fermilab HighBrightness RF Photoinjector", PAC'99, New York, 1999, p. 2027

[10] J.-P. Carneiro, et al, "Study of a Magnetic Chicane at the FNAL/NICADD Photo-injector Using Remote Operation from DESY", EPAC'02, Paris, 2002, p. 1759

[11] N.Barov et al., "Remote Operation of the Fermilab/NICADD Photoinjector", FERMILAB-TM-2167, 2002

[12] http://esgard.lal.in2p3.fr

[13] F.Willeke, private communication 